\title{
Endoscopic Ultrasound Fine-Needle Biopsy May Contribute to the Diagnosis of Malignant Lymph Nodes
}

\author{
Mamoru Takenaka, Shunsuke Omoto and Masatoshi Kudo \\ Department of Gastroenterology and Hepatology, Kindai University Faculty of Medicine, Osaka-Sayama, Japan
}

See "Endoscopic Ultrasound Fine-Needle Aspiration versus Fine-Needle Biopsy for Lymph Node Diagnosis: A Large Multicenter Comparative Analysis” by Diogo Turiani Hourneaux de Moura, Thomas R. McCarty, Pichamol Jirapinyo, et al., on page 600-610.

We would like to comment on a multicenter retrospective study of prospectively collected data for evaluating the outcomes of endoscopic ultrasound fine-needle aspiration (EUSFNA) and endoscopic ultrasound fine-needle biopsy (EUSFNB) for lymph node (LN) sampling authored by de Moura et al. $^{1}$

The treatment strategy and disease prognosis are markedly affected by whether lymphadenopathy is malignant or benign. EUS and EUS-guided sampling are well-known as suitable examination methods for assessing lymphadenopathy. EUS provides a good image of the affected LNs from the lumen of the gastrointestinal tract and allows for tissue sampling using these images for "guidance". EUS-guided sampling has now become the standard of care for close examination of lymphadenopathy. ${ }^{2}$ EUS-FNA is typically performed as EUS-guided sampling, which can provide the LN tissue for cytological evaluation. Since its development, the device has been developed and ingestion about sampling method have been devised to improve the diagnostic efficacy. However, the reported sensitivity and specificity of EUS-FNA ( $88 \%$ and $96.4 \%$, respective-

Received: July 24, 2020 Revised: July 24, 2020

Accepted: July 31, 2020

Correspondence: Mamoru Takenaka

Department of Gastroenterology and Hepatology, Kindai University Faculty of Medicine, 377-2 Ohno-Higashi, Osaka-Sayama 589-8511, Japan

Tel: +81-72-366-0221, Fax: +81-72-367-2880, E-mail: mamoxyo45@gmail.com

ORCID: https://orcid.org/0000-0001-7308-4311

(c) This is an Open Access article distributed under the terms of the Creative Commons Attribution Non-Commercial License (http://creativecommons.org/ licenses/by-nc/3.0) which permits unrestricted non-commercial use, distribution, and reproduction in any medium, provided the original work is properly cited. ly) are less than the ideal values. ${ }^{3}$

Recently, EUS-FNB has been developed as a further development of the EUS-FNA technique. With EUS-FNB, a larger amount of tissue can be sampled compared to that with EUSFNA; this is expected to contribute towards the increase in the efficacy of diagnosing the affected tissue. The usefulness of FNB for subepithelial lesions, pancreatic neuroendocrine tumors, and pancreatic cancer has already been reported. ${ }^{4,5}$ Furthermore, the usefulness of EUS-FNB has also been reported with respect to molecular yields for genomic analysis. ${ }^{5,6}$ In contrast, a comparative study of FNA and FNB for the diagnosis of malignant LNs has not been performed.

When evaluating EUS-FNA or EUS-FNB for the diagnosis of malignant LNs, securing cases with surgical specimens, which are required for pathological analysis, has become difficult, as many cases with malignant LNs are not indicated for surgical resection. Many studies evaluating the LNs have limited their focus to surgical cases. ${ }^{7,8}$

In addition, in many cases, there was a problem with multiple LNs. In these cases, the EUS-FNA results were markedly affected by the lesion targeted for tissue sampling. The usefulness of contrast-enhanced EUS has also been reported, but the identification of appropriate puncture lesions is still challenging. ${ }^{9}$ Moreover, it is very difficult to distinguish between the LNs for which FNA was performed and the resected lesions, even if surgical resection was performed.

In the current issue, the authors performed a multicenter retrospective study to compare the diagnostic efficacy of EUSFNA and EUS-FNB. Postoperative anatomopathological analysis was used as the gold standard for examination in some 
cases. However, as noted by the authors, the gold standard was not the pathological examination of the surgically resected specimens but the patient status after 6 months in many cases. This observation suggests that in many cases, the preoperative and postoperative pathological results were not corroborated on the basis of the specimen pathology. The results of this study should be interpreted with due recognition of this limitation.

In this study, 209 patients underwent EUS-guided LN sampling at five hospitals. The mean lesion size was $16.22 \pm 8.03$ $\mathrm{mm}$, with similar sensitivity and accuracy between FNA and FNB (sensitivity: $67.2 \%$ vs. $75.0 \%, p=0.216$ and accuracy: $78.8 \%$ vs. $83.2 \%, p=0.423$ ). The specificity of FNB was better than that of FNA $(100.0 \%$ vs. $93.6 \%, p=0.01) .{ }^{1}$ Although significant differences were not observed in the diagnostic yields, it was suggested that FNB may have a better clinical effect than FNA.

In addition, the study also included location-specific LN analysis. FNB showed higher sensitivity (FNB vs. FNA: $81.1 \%$ vs. $64.7 \%, p=0.031$ and $80.9 \%$ vs. $58.3 \%, p=0.023)$ and accuracy (FNB vs. FNA: $88.1 \%$ vs. $75.3 \%, p=0.053$ and $88.9 \%$ vs. $70.5 \%, p=0.038$ ) for the abdominal and peri-hepatic LNs, respectively. ${ }^{1}$ These results provide very useful information for daily clinical practice.

The highlight of this paper is that even in situations where rapid on-site evaluation (ROSE) is not possible, a significant difference may not be noted between the diagnostic yield of FNB alone and FNA with ROSE. ROSE allows for earlier diagnosis and a decrease in the number of punctures, and in combination with the large amount of tissue volume obtained with EUS-FNB, it is clearly very useful. ${ }^{10}$ However, ROSE should ideally be performed by a pathologist (although it can also be performed by an endoscopist) and this may not be possible in all institutions.

In the present study, the sampling methods with or without ROSE were also compared. ROSE with FNA showed a higher sensitivity than FNA alone ( $97.7 \%$ vs. $63.5 \%, p=0.004)$. No statistically significant difference was observed between the accuracy of FNA + ROSE and the accuracy of FNB alone (94.4\% vs. $80.7 \%, p=0.161)^{1}$

Although this result should be interpreted with caution, especially since only $17.2 \%$ of the study population underwent additional testing with ROSE according to the author, the results largely support the useful contribution of EUS-FNB in clinical practice. ${ }^{1}$

The authors noted several limitations of their study, including the study design (retrospective design and lack of randomization), selection bias, including different needle sizes, and the possibility that patients were lost to follow-up. However, despite these limitations, this study might shed more light on the difficulties encountered when diagnosing the LNs in clinical practice because this study was the largest multicenter study that specifically compared the effectiveness and safety of EUS-FNA and EUS-FNB for LN sampling.

To confirm the validity of EUS-FNB for LN sampling, further prospective studies including more cases with histopathological answer with surgical specimens under the standardization of needles and ROSE condition are recommended.

Conflicts of Interest

The authors have no financial conflicts of interest.

ORCID

Shunsuke Omoto: https://orcid.org/0000-0001-7291-3608

Masatoshi Kudo: https://orcid.org/0000-0002-4102-3474

\section{REFERENCES}

1. de Moura DTH, McCarty TR, Jirapinyo P, et al. Endoscopic ultrasound fine-needle aspiration versus fine-needle biopsy for lymph node diagnosis: a large multicenter comparative analysis. Clin Endosc 2020;53:600610.

2. Chen VK, Eloubeidi MA. Endoscopic ultrasound-guided fine needle aspiration is superior to lymph node echofeatures: a prospective evaluation of mediastinal and peri-intestinal lymphadenopathy. Am J Gastroenterol 2004;99:628-633.

3. Puli SR, Batapati Krishna Reddy J, Bechtold ML, et al. Endoscopic ultrasound: it's accuracy in evaluating mediastinal lymphadenopathy? A meta-analysis and systematic review. World J Gastroenterol 2008;14:30283037.

4. de Moura DTH, McCarty TR, Jirapinyo P, et al. EUS-guided fine-needle biopsy sampling versus FNA in the diagnosis of subepithelial lesions: a large multicenter study. Gastrointest Endosc 2020;92:108-119.e3.

5. Elhanafi S, Mahmud N, Vergara N, et al. Comparison of endoscopic ultrasound tissue acquisition methods for genomic analysis of pancreatic cancer. J Gastroenterol Hepatol 2019;34:907-913.

6. Asokkumar R, Yung Ka C, Loh T, et al. Comparison of tissue and molecular yield between fine-needle biopsy (FNB) and fine-needle aspiration (FNA): a randomized study. Endosc Int Open 2019;7:E955-E963.

7. Fritscher-Ravens A, Bohuslavizki KH, Brandt L, et al. Mediastinal lymph node involvement in potentially resectable lung cancer: comparison of CT, positron emission tomography, and endoscopic ultrasonography with and without fine-needle aspiration. Chest 2003;123:442-451.

8. Vazquez-Sequeiros E, Norton ID, Clain JE, et al. Impact of EUS-guided fine-needle aspiration on lymph node staging in patients with esophageal carcinoma. Gastrointest Endosc 2001;53:751-757.

9. Miyata T, Kitano M, Omoto S, et al. Contrast-enhanced harmonic endoscopic ultrasonography for assessment of lymph node metastases in pancreatobiliary carcinoma. World J Gastroenterol 2016;22:3381-3391.

10. Iglesias-Garcia J, Lariño-Noia J, Abdulkader I, Domínguez-Muñoz JE. Rapid on-site evaluation of endoscopic-ultrasound-guided fine-needle aspiration diagnosis of pancreatic masses. World J Gastroenterol 2014;20:9451-9457. 